\title{
Application of the TIC's in the design
}

\author{
Olga Elina Feria Ruesga ${ }^{1}$
}

${ }^{1}$ Empresa de Proyectos de Arquitectura e Ingeniería N ${ }^{o} .15$ (EMPROY 15). Santiago de Cuba, Cuba.

Email: oeruesga@emproy15.co.cu

Received: November $27^{\text {th }}, 2017$.

Accepted: January $08^{\text {th }}, 2018$.

Published: March $30^{\text {th }}, 2018$.

Copyright (C2016 by authors and Institute of Technology Galileo of Amazon (ITEGAM).

This work is licensed under the Creative Commons Attribution International

License (CC BY 4.0).

http://creativecommons.org/licenses/by/4.0/

\section{(c) (i) (B) O) Open Aeces:}

\begin{abstract}
The present work is carried out on the operating system Windows, using Microsoft Office ACCESS for the handling of a database (BD) as Agent that gathers relative information to the fields corresponding to the Application of the Services of Design CAD in the Enterprise of Projects (EMPROY 15). The Documental Information is achieved with bigger feasibility, dependability and effectiveness of the process of Administration of all the Digital Information that it is generated in the Services of Design CAD being achieved a saving of time and guaranteeing the continuation of those that are in Execution or Finished obtaining a saving of resources and materials, that which has an economicenvironmental impact.
\end{abstract}

Keywords: Database, Computer system that Negotiates the Information, Computer Systems, Services CAD (Design Attended for Computer) and Information and Communication Technologies (TIC's).

\section{Aplicación de las TIC’s en el Diseño}

\section{RESUMEN}

El presente trabajo se realiza sobre el Sistema Operativo Windows, utilizando Microsoft Office ACCESS para el manejo de una Base de Datos (BD) como Gestor que recopile información relativa a los campos correspondiente a la Solicitud de los Servicios de Diseño CAD en la EMPROY 15.Se logra la Información Documental con mayor factibilidad, confiabilidad y eficacia del proceso de Gestión de toda la Información Digital que se genera en los Servicios de Diseño CAD lográndose un ahorro de tiempo y garantizando la trazabilidad de los que están en Ejecución o Terminados obteniendo un ahorro de recursos y materiales lo cual tiene un impacto económico-ambiental.

Palavras Chaves: Base de Datos, Sistema Informático que Gestione la Información, Sistemas Informáticos, Servicios CAD (Diseño Asistido por Computadora) y Tecnologías de la Información y Comunicación (TIC's).

\section{INTRODUCCIÓN}

Las Tecnologías de la Informatización y las Comunicaciones (TIC's) han permitido que las organizaciones sean más flexibles, reflejado esto en una producción más eficiente, rápida y económica, convirtiéndose éstas en un instrumento cada vez más indispensable que tiene como finalidad elevar la cultura informática, donde pueden realizar múltiples funcionalidades. En el Siglo XXI y ante la presencia de una nueva era donde impera el desarrollo de la Informática, de nuevos hardware y software de mayor prestancia aun cuando muchos países no estén preparados para ello, Cuba, en la actualidad, encara um gran reto de informatizar la sociedad con la perspectiva "integrarse absolutamente a la infraestructura global de información, así como hacer uso óptimo de las nuevas tecnologias" [1].

A fin de alcanzar gran seguridad en el proceso para tratamiento de la información, es necesario la aplicación de los sistemas automatizados de tratamiento de datos, los cuales se caracterizan por prestar gran atención hacia su estructura y almacenamiento; ésta es la premisa por la que trabajan los diseñadores de Sistemas de Gestión de Base de Datos (Sistemas Informáticos). "Esto se explica ya que por médio de las construcciones de estructuras óptimas y por medio de la creación de Sistemas de Gestión de Bases de Datos (SGBD), se puede 
lograr una alta efectividad en el proceso de tratamento de la información" y su diseño.

Particularmente, la EMPROY 15 es la Empresa dedicada al Diseño de Obras de Arquitectura e Ingeniería, Investigaciones, Topografía y Servicios de Dirección y Administración de Inversiones y Obras que tiene como finalidad ofertar servicios competitivos con una alta eficiencia que satisfaga las expectativas de los clientes, cumpliendo con todos los requisitos y legislación vigente reafirmando nuestro liderazgo territorial. Para hacer válida esta política, la organización cuenta con un Sistema de Gestión de la Calidad, Medio Ambiente y de Seguridad y Salud del Trabajo certificados conforme a la NC - ISO 9001: 2008, NC - ISO 14001:2004, NC 18001:2005 y NC- ISO 3001:2007 para asegurar que los niveles de calidad, seguridad y medio ambiente se cumplen y mejoran continuamente, fijando objetivos y metas que permitan el cumplimiento de esta política.

Con relación a lo anterior, resulta imprescindible que todos los datos, elementos y estudios que puedan orientar, divulgar y brindar conocimientos a todos los usuarios inmersos en el proceso de diseño, se encuentren en un estado y manera que logren adaptarse a los nuevos cambios que están ocurriendo; es decir, archivar, almacenar y resguardar toda la información de manera digital, de forma tal que pueda ser fácil acceder a la misma, con seguridad, fiabilidad y rapidez, logrando mantener de esta forma una trazabilidad acorde a la calidad del produto que se elabora digitalmente en los Servicios CAD (Diseño Asistido por Computadora) referido a toda la documentación gráfica, textos, cálculos, etc., para obtener una base documental y de consulta por los Usuarios (Clientes Internos o Externos), a los Registros de todos los Servicios que se desarrollan dentro de la entidad desde que comienza en el proceso de Contratación hasta la entrega al Inversionista y la salva definitiva.

\section{DESARROLLO}

La Informatización de los Proyectos de Arquitectura, Ingeniería e Industriales es una innovación de la tecnología de proyección, que origina una forma de actuación laboral y um nuevo pensamiento profesional para la Gestión y toma de decisiones en la tecnología e innovación que permite generar nuevas estrategias y mejoras en los procesos para el desarrollo de la entidad y fortalecer el conocimiento.

Esta Base de Datos (BD) está ubicada en el Servidor principal de la Empresa que tiene instalado como Sistema Operativo Windows Server 2000 con 16 GB de RAM lo que permite su acceso desde cualquier terminal de la red utilizando Microsoft Office ACCESS para el diseño de la misma que durante el proceso de Gestión de la información de los Servicios de Diseño CAD en la EMPROY 15 garantiza la trazabilidad del producto, fiabilidad en los resultados y fácil localización, el acceso es limitado de solo lectura para los Usuarios de la red y total para los Administradores.

La BD se realiza sobre Plataformas Propietarias y actualmente se trabaja como se refleja en la Recomendaciones llevarlo a MSQL.

En base a lo antes analizado se define como el problema, Insuficiente Aplicación de las TIC's en la Gestión de la Información Documental de los Servicios CAD EMRPOY 15.

El Objeto el Sistema Informático que Gestione la Información Documental de los Servicios CAD en la EMRPOY 15 .
El Objeto General evaluar la Aplicación de las TIC's en el Diseño de la Gestión de la Información Documental de los Servicios CAD EMRPOY 15.

El Objetivo Específico evaluar rapidez de acceso y trazabilidad en la Gestión de la Información Documental de los Servicios CAD con la Aplicación de las Tecnologías de la Información y Comunicación (TIC's) en el Diseño en la EMRPOY 15.

El campo de acción, el Sistema que Gestione la Información Documental de los Servicios ejecutados de Diseño en la EMPROY 15.

Hipótesis:

- Si se implementa Sistema de Gestión de la Información de los Servicios CAD se facilitará la gestión de la información documental con mayor factibilidad, confiabilidad y eficacia em el momento preciso, disminuyendo las insuficiencias en dicho proceso.

Para el desarrollo del Sistema Informático se desarrollaron las siguientes tarefas:

1. Diagnóstico.

2. Caracterizar históricamente, conceptualmente y en la actualidad, un Sistema Informático que Gestione la Información Documental de los Servicios CAD en la EMPROY 15.

3. Caracterizar el Sistema Informático que Gestione la Información Documental de los Servicios.

4. Desarrollar un Sistema Informático que Gestione la Información Documental de los Servicios en la EMPROY 15.

5. Evaluar el Sistema Informático propuesto a partir de las valoraciones de Especialistas en la EMPROY 15.

Técnicas y métodos empleados en la investigación:

- Análisis documental

- Observación

- Entrevistas

- Histórico Lógico

- Análisis y Síntesis

- Diseño y programación.

El aporte práctico de la investigación está dado en um sistema informático que gestione la información existente em la EMPROY 15 de Santiago de Cuba.

Significación práctica de la investigación el sistema informático propuesto contribuye a lograr uma mayor eficiencia, especificidad, facilidad y seguridad al analizar toda la información existente.

Con esta automatización se crean las bases de datos para posteriormente afrontar mayores empeños en cuanto a la informatización de los procesos en los Servicios CAD, lo que redundará en una gestión más confiable en todo el proceso y su perfeccionamiento constante.

El sistema propuesto puede ser aplicable a otras empresas similares que tengan implantado el Sistema de Gestión Integrado de Calidad, lo que posibilitaría una plataforma eficiente y confiable además propiciará la capacitación a estudiantes y adiestrados así como al personal que trabaja directamente con los proyectos que se ejecutan en la empresa.

El principal rasgo distintivo de dicho sistema es el hecho de que por primera vez se realiza un Sistema Informático que 
Gestione la Información Documental de los Servicios que garantice los fines antes expuestos.

\section{II.1. PROCESO DE SISTEMA INFORMÁTICO QUE GESTIONE LA INFORMACIÓN DOCUMENTAL DE LOS SERVICIOS CAD}

II.1.1. CARACTERIZACIÓN DESDE EL PUNTO DE VISTA CONCEPTUAL DEL PROCESO DE SISTEMA INFORMÁTICO QUE GESTIONE LA INFORMACIÓN DOCUMENTAL DE LOS SERVICIOS CAD EN LA EMPROY 15

\section{II.1.1.1. BREVE DESCRIPCIÓN DE LA EMPROY 15}

La EMPROY 15 es una entidad dentro del Grupo Empresarial dedicada al Diseño de Obras de Arquitectura em Ingeniería, Investigaciones, Topografía y Servicios de Dirección y Administración de Inversiones y Obras.

En dicha empresa se identifican dos procesos:

- Proceso principal: Son todos los procesos asociados al servicio principal que desarrolla la entidad.

- Proceso de apoyo: Son todos los procesos que tributan a los servicios principales.

\section{1.1.2. SERVICIOS CAD}

Los servicios CAD se encuentran enmarcados dentro de la Gestión de Desarrollo y responde a la actividad del processo desde que entra el servicio (proyecto) por el Inversionista a la Dirección de Negocios hasta que se le entrega la documentación al mismo en formato digital o impreso.

Control de las actividades en los Servicios CAD en los Servicios CAD intervienen varias áreas en correspondência con la estructura de la entidad y los niveles de jerarquía que se tienen implantados, por lo que cada una le corresponde uma actividad determinada y una responsabilidad diferente.

\section{II.1.1.3. SISTEMAS INFORMÁTICOS}

Un sistema informático es el conjunto de hardware, software y de un soporte humano; éste se vale de una computadora y ésta a su vez suele estar formada por una unidad central de proceso (CPU de sus siglas en inglés Central Procesing Unit), dispositivos de entrada, dispositivos de almacenamiento y dispositivos de salida. La CPU incluye una unidad aritméticológica (ALU), registros, sección de control y bus lógico. La unidad aritmético - lógica efectúa las operaciones aritméticas y lógicas.

Los registros almacenan los datos y los resultados de las operaciones. La unidad de control regula y controla diversas operaciones. El bus interno conecta las unidades de la CPU entre sí y con los componentes externos del sistema. En la mayoría de las computadoras, el principal dispositivo de entrada es el teclado. Dispositivos de almacenamiento son los discos duros, flexibles (disquetes) y compactos (CD).

Dispositivos de salida que permiten ver los datos son los monitores e impresoras.

Principales elementos que forman parte de un sistema informático:

- Software.

- Hardware.
- Persona.

- Base de Datos.

- Documentación.

\section{II.1.1.4. ANÁLISIS Y DISEÑO DEL SISTEMA INFORMÁTICO}

El análisis y diseño de Sistema Informático es el proceso de estudio de la situación de una organización o empresa, con la finalidad de observar cómo trabaja y decidir si es necessário realizar una mejora.

El proceso de creación de un sistema informático consta de un conjunto de tareas diferentes de forma secuencial. Las etapas de este proceso son las siguientes:

- Reconocimiento de la necesidad de cambios

- Estudio de la factibilidad técnico económica

- Análisis detallado de la situación actual

- Diseño y desarrollo.

- Implantación y prueba del sistema.

- Implantación definitiva.

- Perfeccionamiento del sistema.

En la actualidad existen varios SGBD entre los que pueden mencionarse: Oracle, SQL Server, MySQL, Postgresql, FoxPro, Clipper, dBase y Microsoft Access, entre otros.

En esta investigación se selecciona para el trabajo el Microsoft Access por las características de programación visual, así como las facilidades que presenta en la construcción de Consultas, Formularios e Informes ganando en eficiencia en la elaboración del producto.

Las características principales del Microsoft Access son las siguientes:

- Incorpora características nuevas para facilitar el diseño y uso de bases de datos y hacerlas más potentes.

- Posibilita la creación de bases de datos con la ayuda del Asistente para bases de datos, lo cual le sirve al programador para hacer rápidamente sus bases de datos partiendo de muchas plantillas.

- Facilita la optimización del rendimiento de las bases de datos; utilizando el Analizador.

- Establece las propiedades de la base de datos, dando la comodidad de que el programador pueda ver, cambiar y definir las propiedades de la base de datos.

- Se pueden crear aplicaciones personalizadas facilmente usando las nuevas propiedades de inicio para especificar si se muestra automáticamente un formulario determinado al abrir la base de datos o controlar si se muestran los menús personalizados o los incorporados, la ventana de la base de datos o la barra de estado.

\section{II.1.2. CARACTERIZACIÓN HISTÓRICA DEL PROCESO DE LA INFORMACIÓN DOCUMENTAL DE LOS SERVICIOS CAD EN LA EMPROY 15}

La EMPROY 15 fue fundada en el año 1975. El sistema de perfeccionamiento es aprobado en el año 2002 y el Sistema de Gestión Integrado de la Calidad se certifica por primera vez em el año 2002. A finales de Septiembre de 2001 que se estabelece oficialmente el Procedimiento PEC 06. A1. SOLICITUD DEL SERVICIO CAD PARA SERVICIOS DE DISEÑO. 
II.2. CARACTERIZACIÓN DEL ACTUAL PROCESO DEL SISTEMA INFORMÁTICO QUE GESTIONE LA INFORMACIÓN DOCUMENTAL DE LOS SERVICIOS CAD EN LA EMPROY 15

Actualmente los Servicios CAD en la EMPROY 15, es llevado en los Registros plenamente identificados en el proceso de Gestión de la Calidad, una vez que este proyecto sale de ejecución o es declarado como paralizado se procede a la salva definitiva en CD/DVD. La solicitud del Servicio CAD, es realizada personalmente por el Usuario autorizado según está descrito.

El Registro es llenado manualmente donde aparece detallado todos los datos necesarios para identificar un Servicio de Diseño determinado especificando los Sistemas, Sub Sistemas y Especialidades por cada uno conjuntamente con el equipo de trabajo.

Cuando es necesario realizar alguna modificación ésta tiene que ser solicitada en el Registro con fecha y firma de cuando se realiza, especificando el tipo de la misma, es decir:

- Cambio del Proyectista General o Proyectista Responsable. Cambio del Jefe del Taller de Diseño.

- Aumentar o quitar un Sistema o Sub Sistema.

- Aumentar o quitar una especialidad determinada.

- Aumentar o quitar a uno o varios miembros del Equipo de Trabajo.

- Cambio del Código del Proyecto.

- Cambio del nombre del Fichero.

Estos registros tienen que ser guardados de por 5 años para que exista la evidencia de los proyectos en los que se há trabajado así como el contenido de los mismos.

De lo anterior se evidencia que lo que se tiene estabelecido actualmente trae algunas desventajas en cuanto al correcto funcionamiento de un proceso dentro de la entidad y el constante movimiento que se genera en búsqueda de información o datos de los proyectos, creando esto moléstias por pérdida de tiempo con relación a la generación de servicios con una alta competitividad, fiabilidad y fluidez em los mismos, lo cual constituye la razón de ser de la entidad.

Por otra parte, existe un movimiento constante de forma general del Cliente interno o externo, para consultar cualquier información o dato del Proyecto, ya sea, de la información que está en ejecución en esos momentos o la que se tiene almacenada en CD/DVD como ya terminada.

De lo planteado anteriormente se evidencia la necesidad de resolver tal situación, siendo una de las vías, la creación de una Base de Datos estructurada correctamente teniendo em cuenta la Tecnología de la Informatización y las Comunicaciones (TIC's) con la cual se podría alcanzar um adecuado manejo de toda la información que se elabora digitalmente, una vez identificados los problemas actuales y sus posibles mejoras.

\section{SISTEMA INFORMÁTICO DE GESTIÓN DE LOS SERVICIOS EXISTENTE EN LA EMRPOY 15}

A continuación se realiza una descripción del Sistema Informático durante el desarrollo de la propuesta del software empleado que conducen a la obtención de un produto terminado acorde al problema planteado inicialmente para ser empleado directamente en el proceso productivo de nuestra empresa.

\section{III.1. CAPA DE DATOS}

Valiéndose de Microsoft Access, se logra disponer de toda la información desde un único archivo de base de datos. Dentro del registro, se alcanza fragmentar los datos en contenedores de almacenamiento independientes llamados tablas; puede ver, añadir y renovar datos de la tabla por medio de formularios; consigue además, buscar y rescatar sólo los datos que deseé por medio de consultas; y puede examinar o imprimir datos con un diseño específico por medio de informes.

\section{III.2. ANÁLISIS DE DATOS}

A toda la información y los datos recopilados sobre los Registros de Solicitud CAD y los Procedimientos vigentes acordes al Sistema Integrado de Gestión implantado, se le realizó un análisis que permitió clasificar los Servicios que se ejecutan de acuerdo a las características del sistema, desde el comienzo del producto, su tiempo de ejecución y grado de protección, creando un inventario físico que soportaría la información en cuanto a solicitudes, modificaciones, validaciones y salva en CD/DVD en correspondencia a la estructura de las diferentes Direcciones de Proyecto.

\section{III.2.1. DISEÑO DE LA BASE DE DATOS}

A continuación se muestra el Diagrama Entidad-Relación (DER) que recoge toda la semántica del problema tomando em consideración los requisitos del cliente, así como las restricciones impuestas al flujo de información documental que ejecuta en la EMRPOY 15. Figura 1 (a) Diagrama Entidad - Relación del diseño de la Base de Datos.

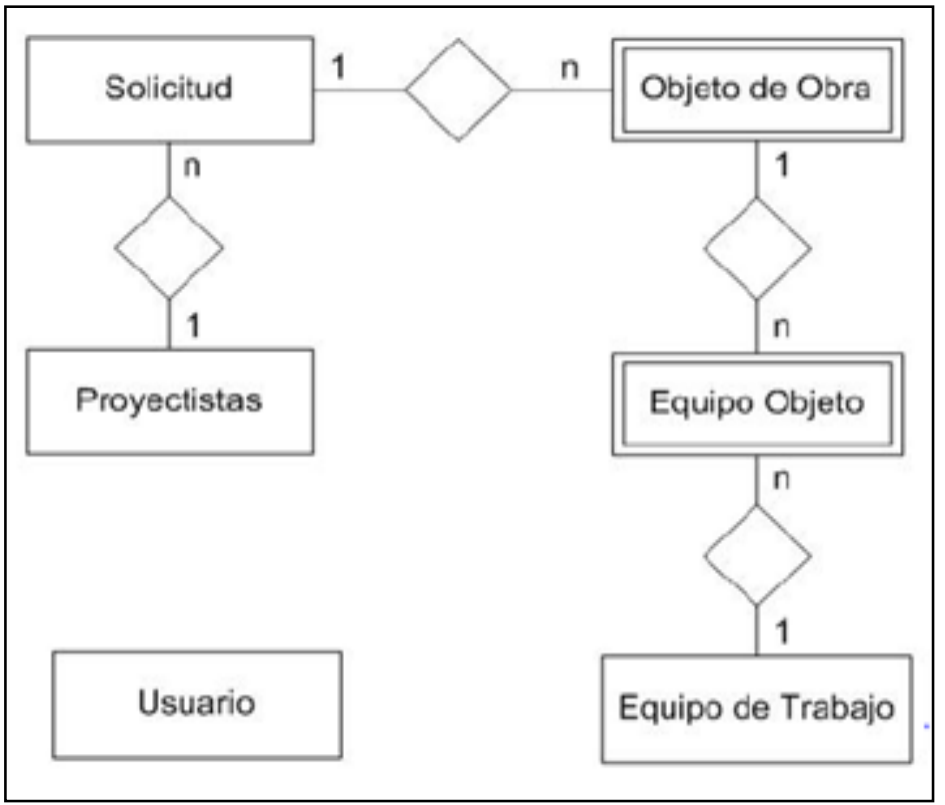

Figura 1: Diagrama Entidad - Relación del diseño de la Base de Datos.

Fuente: Autor, (2018).

En el proceso de transformación del DER a tablas quedó el esquema de base de datos siguiente:

- Usuario (Usuario, Contraseña, Roles, Activo) Proyectistas (IdProyectista, NombreProyec) EquipoDeTrab (IdNombre, NombreEqTrab) EquipoObjeto (IdEqTrab, IdProyecto, NombreObjeto, Fecha, FechaEquipo) Solicitud (IdProyecto, 
NombProyecto, Código, Fichero, ...) ObjetosDeObra (IdProyecto, NombreObjeto, Fecha, Sist, Subsist, PG, AR, CD). Posteriormente se normalizaron cada una de las tablas de la base de datos hasta la Forma Normal de Boyce-Codd, por lo tanto, se garantiza que cumple con las formas normales anteriores (1FN, $2 \mathrm{FN}$ y $3 \mathrm{FN}$ ), de modo que se asegura que el determinante es una superllave, se eliminaron las dependencias transitivas entre atributos no llaves, se eliminaron las dependencias parciales con respecto a la llave, se eliminaron los grupos repetitivos y se asevera la atomicidad de los atributos.

Realizado todo este proceso se puede afirmar que el diseño de la base de datos se encuentra en Forma Normal de BoyceCodd.

\section{III.3. CREACIÓN DE LOS OBJETOS DE LA BASE DE DATOS}

Una base de datos es una serie de datos organizados y relacionados entre sí, los cuales son recolectados y explotados por los sistemas de información de una empresa, es el conjunto de todas las Tablas y otros objetos (tales como: Consultas, Formularios, Informes, Macros y Módulos) que se utilizan para gestionar datos.

\section{II.3.1. CREACIÓN DE LAS TABLAS}

Mediante la utilización del asistente que brinda Microsoft Access o haciendo uso del modo diseño, se especifica cada uno de los campos que conformará la tabla, así como cada uno de los tipos de datos correspondientes. Figura 2 (b) Ejemplo de Tabla de Solicitud.

\begin{tabular}{|c|c|c|c|}
\hline \multicolumn{4}{|c|}{ ESolikituds: Tabla } \\
\hline & bakedering & fogenger & \\
\hline 8 & Srovede & Atowrienco & \\
\hline & Nenbioyeds & fete & tortus del Avecto \\
\hline & Colop & lete & codecowhorecte \\
\hline & Evets & tete & 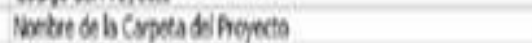 \\
\hline & Whe & tete & lortud difders bh heyste \\
\hline & is rovetists & teito & 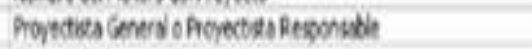 \\
\hline Ot & erovile & 800 & Peosclihopde \\
\hline & atesth & saso & Exos del hoyedo \\
\hline & Bacati & Savo & Exodihosedo \\
\hline 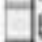 & Bucall & Sing & Dropdothonde \\
\hline & tenses & $\sin$ & tros di howedo \\
\hline & towant & sen & Daos cel horedo \\
\hline & Gouto & Nien & j'ter datesto \\
\hline 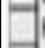 & Fechosiland & fechuten & 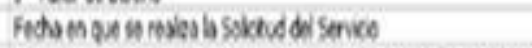 \\
\hline 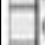 & Coiensostes & Nent & 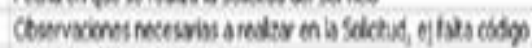 \\
\hline & thab & lete & 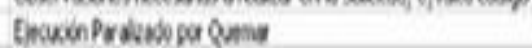 \\
\hline F & inte & sons & Uso de Bderenos biteny \\
\hline & fechniduro & Fochapias & Pedangen us swas CD \\
\hline & wace & lete & uread ded \\
\hline
\end{tabular}

Figura 2: Ejemplo de Tabla de Solicitud.

Fuente: Autor, (2018).

\section{III.3.2. CREACIÓN DE LAS RELACIONES ENTRE LAS TABLAS}

Después de haber creado todas las tablas que componen la base de datos es necesario crear las relaciones que existen entre ellas. Para este fin se toma en cuenta el DER empleado en el diseño de la base de datos, así como los requerimientos del cliente.

A continuación se muestra la implementación de las relaciones entre las tablas en Microsoft Access. Figura 3 (c) Relaciones de la BD en Microsoft Access.

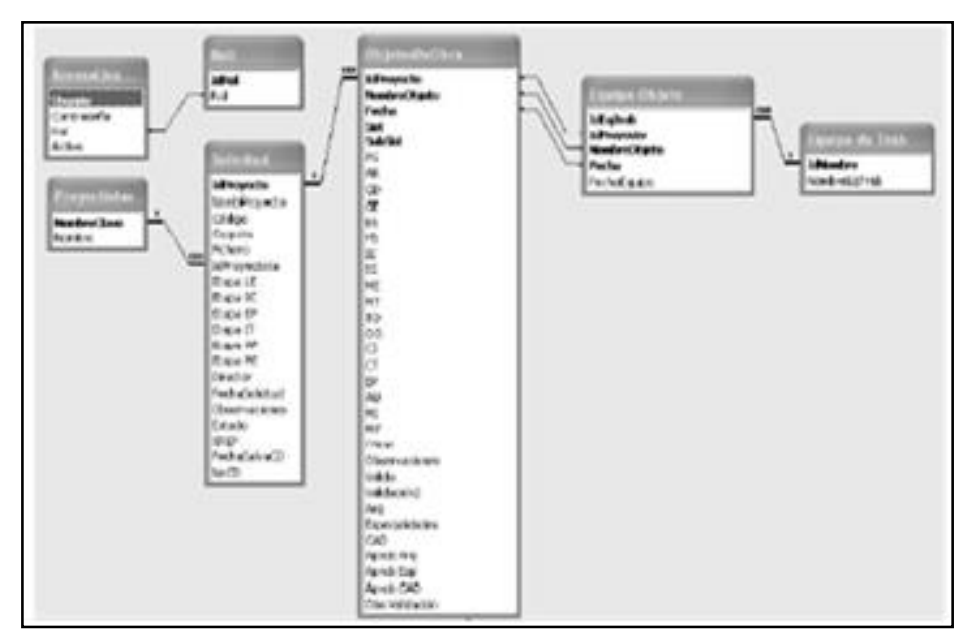

Figura 3: Relaciones de la BD en Microsoft Access.

Fuente: Autor, (2018).

\section{III.3.3. CREACIÓN DE LOS FORMULARIOS}

Permite mostrar e introducir los datos de forma más cómoda y visiblemente más atractiva. Para crearlos normalmente se utiliza el asistente y luego en vista de diseño es personalizado.

\section{III.3.4. CREACIÓN DE LAS CONSULTAS}

Es una herramienta para hacer preguntas y realizar acciones sobre los datos en las tablas. Además permite gestionar grandes cantidades de datos. La vista de diseño fue el estilo de trabajo fundamental en la creación de las consultas.

\section{III.3.5. CREACIÓN DE LOS INFORMES}

Permiten imprimir o ver previamente los datos de forma personalizada. Para su elaboración se utilizo fundamentalmente el asistente y posteriormente la vista diseño para personalizarlo según los intereses del cliente.

\section{III.3.6. CREACIÓN DE LOS MÓDULOS}

Mediante la programación en Visual Basic se elaboraron funciones y procedimientos que complementaron las funcionalidades que tiene el sistema, a continuación se mencionan los siguientes:

- Procedimiento GeneraArbolCarpeta: Utilizado para generar la estructura del árbol de carpetas, teniendo en cuenta el nombre del proyecto especificado en el campo carpeta a partir del cual se genera el sistema, sub sistema, especialidades y subcarpetas por cada especialidad según la solicitud realizada.

- Función DevSemestre: Devuelve el número del Semestre dada una fecha.

- Función DevTrimestre: Devuelve el número del Trimestre dada una fecha.

\section{III.4. DESCRIPCIÓN DEL SISTEMA INFORMÁTICO}

El sistema tiene el objetivo de sustituir los Registros del Servicio CAD que se llevan manualmente por un sistema informático que permita mantener actualizado toda la información que se procesa con la celeridad que requiere, disponer de un 
mayor control de la información de los servicios que se ejecutan, teniendo en cuenta un mayor nível de información y actualización del estado en que se encuentran los Servicios en ejecución o están terminados.

Todo esto permite una mayor relación hombre máquina en la informatización de los procesos, así como en el uso de la INTRANET y el correo electrónico.

\section{III.5. FUNCIONAMIENTO DEL SISTEMA}

Al ejecutar el sistema este se inicia con el siguiente formulario. Figura 4 (d) Formulario de acceso.

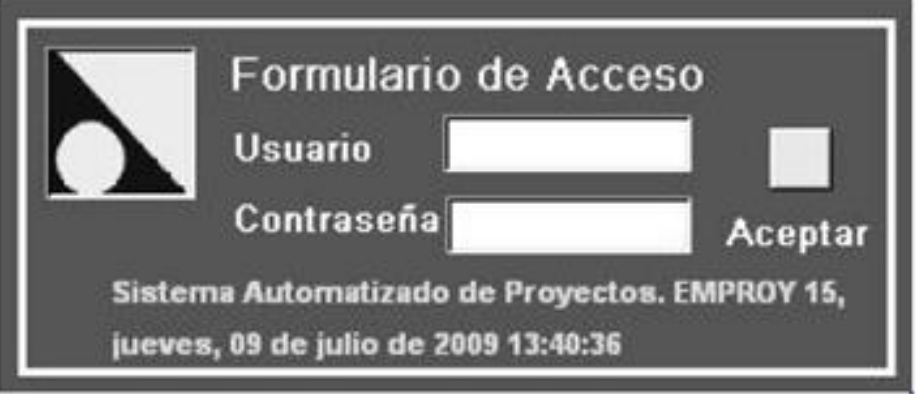

Figura 4: Formulario de acceso.

Fuente: Autor, (2018).

El "Formulário de Accesso" solicita el nombre del usuario y contraseña, información que se valida en una tabla que recoge estos datos, que a su vez contiene el rol asignado a cada usuario (administrador, usuario avanzado o usuario) determinándose de esta forma a qué funcionalidades tiene acceso en el sistema.

Una vez realizada la validación se muestra el formulario principal del Servicio CAD donde aparecen las opciones siguientes: Figura 5 (e) Menú Principal.

Es válido señalar que esta BD se trabajó hasta el 2009 con campos que no brindan toda la información importante a tener de un Proyecto o Servicio y como acción de mejora y ante los inconvenientes que confrontamos respecto a que los Registros de la Solicitudes CAD se conservan solo por 5 años que esto trae aparejado que al desaparecer los mismos se pierde la trazabilidad de los Proyectos provoca modificar la misma al crear nuevos campos.

\section{III.5.1. MENÚ PRINCIPAL}

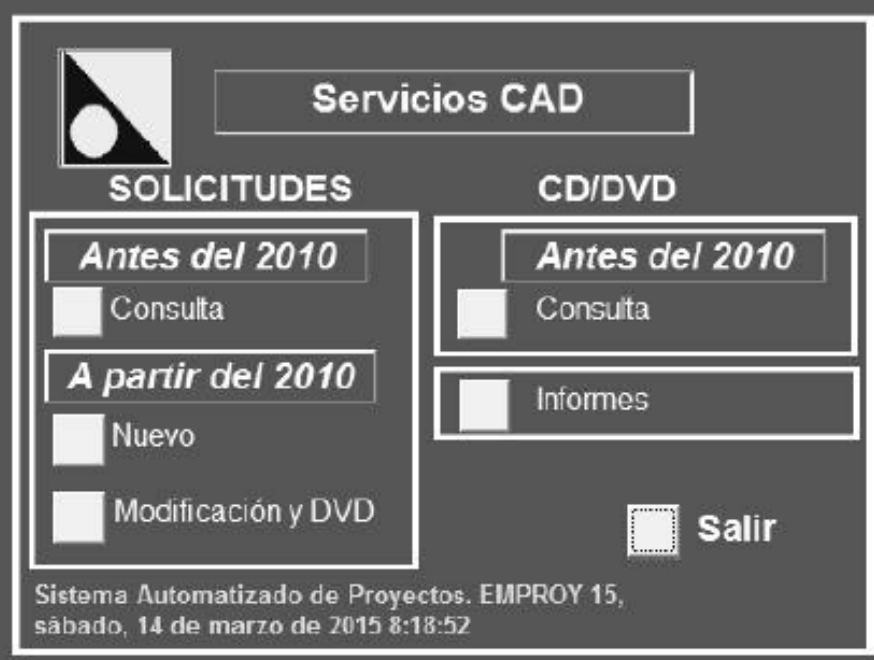

Figura 5: Menú Principal.

Fuente: Autor, (2018).
En el Menú Principal se observa que está dividido em Servicios antes del 2010 y a partir del 2010 lo cual aparece fundamentado anteriormente.

\section{III.5.1.1. VISUALIZAR SOLICITUDES SERVICIOS CAD ANTES DEL 2010}

La selección de esta opción carga el formato "Solicitud del Serviço CAD". El mismo permite a los usuários consultar todo lo correspondiente a los campos declarados, ej. Nombre del Proyecto, Código. Proyectista General o Responsable y otros dándole información si están en Ejecución, en fase de quema (para Auditar) o bien Paralizado, por otra parte, les aportan datos a los usuarios para la organización de los ficheros dentro de la carpeta del proyecto. También cuenta com la posibilidad de enviar por correo electrónico alguna consulta de un proyecto determinado.

Todos los campos de este formulario se encuentran bloqueados para impedir que cualquier dato sea modificado.

\section{III.5.1.2. SERVICIOS ANTES DE 2010 EN CD/DVD}

Al cargar el olicitud Servicio CAD Antes del Con solo introducir un campo de igual forma descrito anteriormente localizamos rápidamente en que $\mathrm{CD} / \mathrm{DVD}$ se encuentra permitiendo que el Usuario solicite el mismo y a través de la red acceda a buscar la información deseada.

\section{III.5.2. NUEVA SOLICITUD DEL SERVICIO CAD}

Cuando se solicita un nuevo Servicio por parte del Director de Proyecto, el Proyectista General o Proyectista Responsable, aparecen nuevos campos que no se contemplaban en la de antes de 2010 como anteriormente se explica.

Una vez introducido este Servicio pasamos a crear un *.pdf y guardarlo en la Carpeta correspondiente al Director de Proyecto con el objetivo de que cada Especialista que interviene en el mismo conozca los campos necesarios al nombrar el Fichero cosa esta que esta descrita en el Procedimiento SOLICITUD DEL SERVICIO CAD PARA SERVICIOS DE DISEÑO. PEC.06.A1.

\section{III.5.3. MODIFICACIÓN Y CD/DVD A PARTIR DE 2010}

A partir de estos Formularios y con el objetivo que creamos esta modificación en la BD es que se notan los câmbios fundamentado en las acciones de mejora en busca de identificar lo que realmente además de lo que teníamos debíamos controlar. En este caso se comporta de forma diferente a las del antes de 2010 ya que nos brinda más información por ej. Objetos de Obra, Especialidades y Equipo de Proyectos Solicitado cosa esta importante para mantener una trazabilidad del Servicio. Figura 6 (f) Modifica el Servicio CAD salvados a partir de 2010. 
Ruesga, ITEGAM-JETIA. Vol. 04, № 13, pp 36-43. March, 2018.

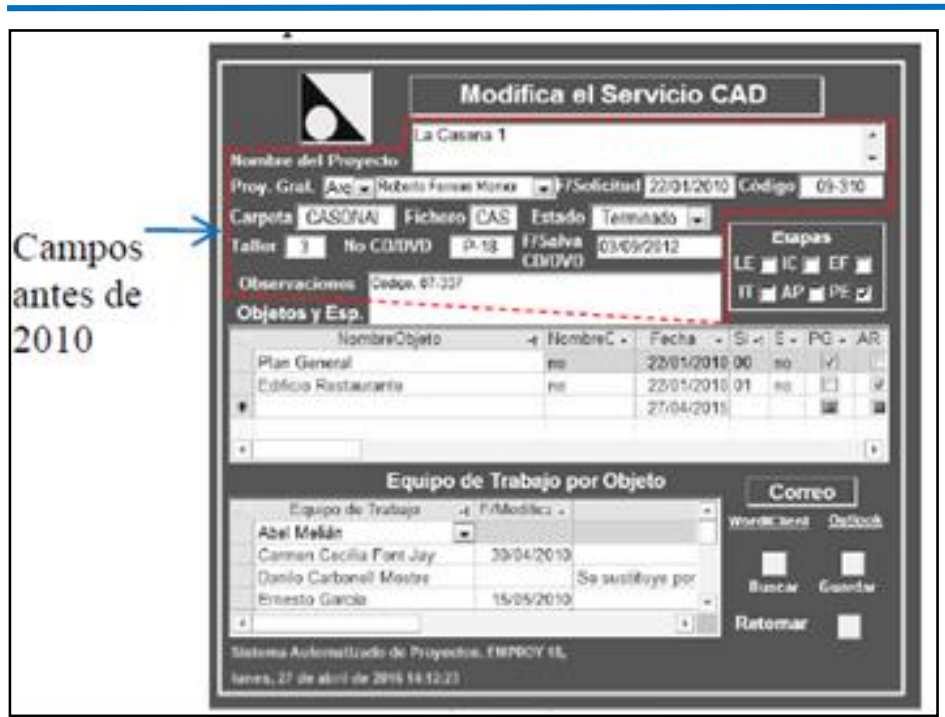

Figura 6: Modifica el Servicio CAD salvados a partir de 2010. Fuente: Autor, (2018).

El resto de los Campos no contenidos dentro de los limites señalados corresponde a los creados.

En esta ventana como su nombre lo identifica nos permite realizar modificaciones en lo correspondiente a aumentar Objetos de Obra, Especialidades y Equipo de Trabajo, solamente por los autorizados, además ya no es necessário consultar otro Formulario para ver si está en CD/DVD ya que automáticamente nos muestra en cual esta y en caso que salga por cualquier Modificación aparece en las Observaciones.

\section{III.5.4. INFORMES}

Muestra todo lo que hasta el momento necesitamos para los procesos que llevamos dentro del sistema Informativo. Figura 7 (g) Informes.

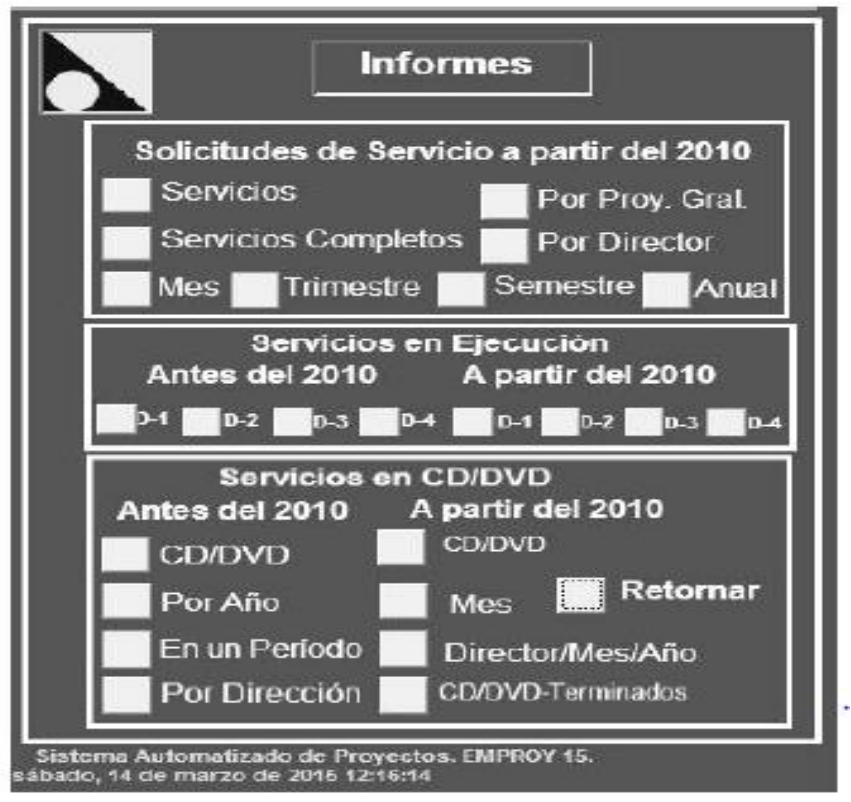

Figura 7: Informes.

Fuente: Autor, (2018).

Muestra todos los Informes que se necesitan para el Sistema Informativo y los Procesos a partir de 2010. Figura 8 (k) Ejemplo de un Informe.

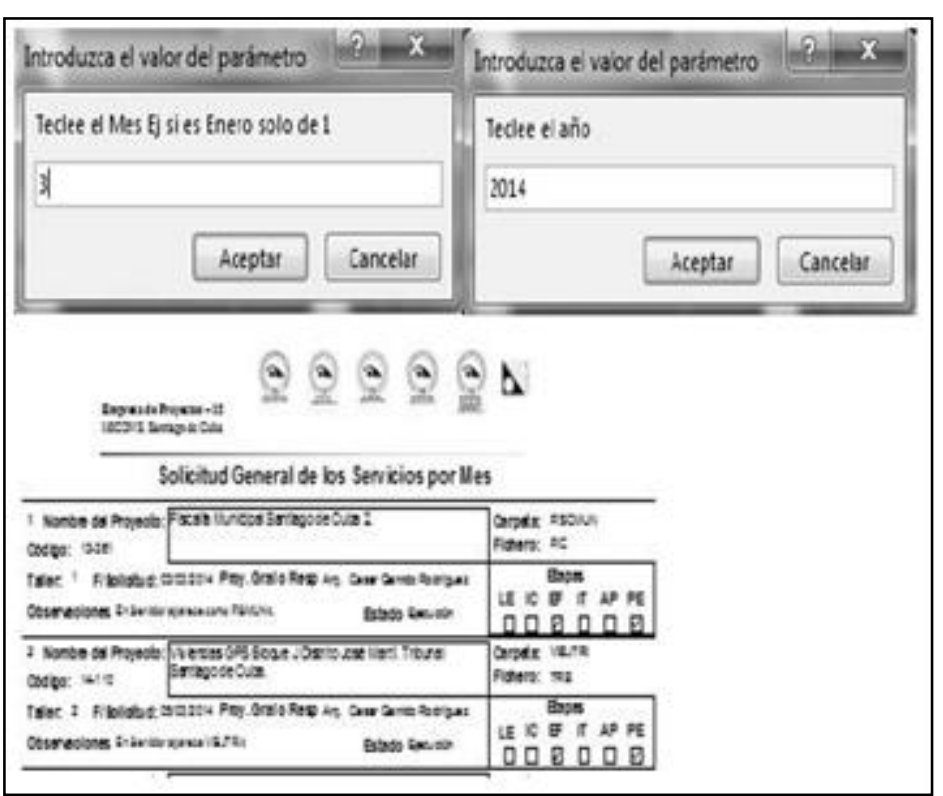

Figura 8: Ejemplo de un Informe.

Fuente: Autor, (2018).

En este caso se escoge Solicitud de Servicio a partir de 2010 por Mes, la cual nos da la cantidad de Servicios solicitados por Mes y Año. De esta misma forma nos dará según el Informe que se desee.

\section{IMPACTO ECONÓMICO}

Es difícil realizar un cálculo de lo que se ahorra com Aplicación de las TIC's em el diseño de la Gestión de la Información Documental de los Servicios CAD EMRPOY 15 sin embargo me referiré solamente al ahorro de papel sin contar el tiempo que el solicitante del Servicio utiliza en llenar el mismo y moverse de su puesto de trabajo ya actualmente como acción de mejora se hace la Solicitud por correo.

Se parte de 1 Paquete trae 200 hojas.

Tabla 1: cálculo de gastos.

\begin{tabular}{ccc}
\hline Registros & Costo CUC & Gasto \\
\hline 4600 & 15.25 & 350.75 \\
\hline Registros & Costo MC & Gasto \\
\hline 4600 & 19.75 & 454.25 \\
\hline
\end{tabular}

Fuente: Autor, (2018).

Se evidencia el ahorro con esta acción tomada al igual que se guarda como evidencia en la Carpeta de cada Servicio mientras está en Ejecución o ya al ser Terminado y el Registro llevado manualmente desaparece a los 5 Años.

El presente trabajo se encuentra en el Servidor de la Empresa que tiene como Sistema Operativo Windows Server 2000 com 16 GB de RAM lo que permite revisar o consultar cualquier Servicio de Diseño en qué estado está en el momento que lo necesitan, permitiendo una eficiencia, fiabilidad, ahorro de tiempo de trabajo y eficacia de la Información Documental ya que cada Usuario de la red tiene en su escritorio un acceso directo y es compatible con cualquier versión de Office, siendo el uso de memoria de la PC ínfimo, evitando su movimiento a otro puesto de trabajo. 


\section{CONCLUSIONES}

El sistema informático facilita la gestión de la información documental con mayor factibilidad, confiabilidad y eficacia em el momento preciso, disminuyendo las insuficiencias en dicho proceso.

La informatización de todas las actividades que desarrolla y su proceso principal, ha contribuido a elevar sostenidamente sus indicadores de gestión económica y de recursos em general, de igual forma esto ha requerido una mayor organización de la información para su rápida identificación y trazabilidad.

Ahorrar los recursos puestos a nuestra disposición para mitigar el impacto que sobre el medio ambiente tiene el desarrollo de los procesos, elevar la calidad de la información, mejorar los métodos de trabajo, aumentar la eficacia y eficiencia en el tiempo dedicado por el proyectista al servicio, contribuir a la disminución de los plazos de ejecución del proyecto y aumentar la productividad fueron los criterios y necesidades de la entidad tenidos en cuenta para desarrollar el trabajo, lo que evidencia que como solución computacional es efectiva ya no se contaba con ninguna.

Esta propuesta se ha convertido en un magnifico instrumento en manos de los Usuarios para el desarrollo de una mejor gestión que redunda en la satisfacción de nuestros clientes Internos y Externos.

\section{RECOMENDACIONES}

Implementar el Sistema Informático en un servidor SQL con el objetivo de lograr una mayor eficiencia en la concurrencia, seguridad y usos de los recursos que proporciona el sistema de gestión de base de datos Microsoft SQL Server.

\section{AGRADECIMIENTO}

Mi agradecimiento va dirigido al apoyo brindado por mi esposo y en especial a la EMPROY 15.

\section{REFERENCIAS}

[1] Colectivo de Autores, Informática Médica. Editorial Ciencias Médicas, 1999. Tomo 1 pp 16-77 ISBN 959-7132-06-0. Cuba.

[2] C. N. Prague, W.C. Amo and J.D. Foxall. Los Secretos de Access 97.Editorial Anaya Multimedia. 01/01/1997, pp. 25-48, 2010 .

[3] M. Castelles. El impacto de las nuevas tecnologías en la economia internacional: implicaciones para la economía española, Madrid: Instituto de Estudios de Prospectiva. 1990, p.p. 461-491.

[4] M. Castelles. La ciudad informacional. Tecnologías de la información estructuración económica y el proceso urbanoregional, Madrid: Alianza Editorial, 1995, p.p. 125-129.

[5] A. Simpson, and E. Olson. La Biblia del Access 97, Editorial: Anaya Multimedia, Madrid 1997, p.p. 233-240.

[6] PEC. 06. Organización de los Proyectos Digitalizados. EMPROY 15. Feria, OE, 2007.
[7] PEC.06.A1. Solicitud CAD para Servicios de Diseño. EMPROY 15. Feria, OE, 2007.

[8] T. García. Los Sistemas de Información en las Organizaciones. Oct. 10-15, 2002, Cuba.

[9] V. Muñiz, "Estrategia de Superación, uma alternativa para elevar la preparación del instructor de los Joven Club de Computación y Electrónica" Dept. Elect. Universidad de Oriente, Cuba, 2007.

[10] S. Caraballo, and R. Cicala. (2010, May. 5). "Hacia uma didáctica de la Informática, Available in: http://www.epi.asso.fr/revue/articles/a0601c_esp.htm

[11] I. Cisneros, and C. García ," ? Sociedad de la información, ? Sociedad del conocimento?". Available in: http://tecnologiaedu.us.es/edutec/paginas/43.htm.

[12] D. Pérez. (2007, Oct. 26). "?Qué son las bases de datos", Available in: http://www.maestrosdelweb.com/que-son-las-basesde-datos.

[13] ohamygood (2010, Jun. 17). Foro todoexpertos "Control de

Available

in:

http://www.todoexpertos.com/categorias/tecnologia-e-

internet/bases-de-datos/microsoft-

access/respuestas/173814/control-de-usuarios.

[14] ainhi (2003, Mar.22). Foro forosdelweb "Conectar a una base de datos access desde visual basic". Available in: http://www.forosdelweb.com/f69/conectar-base-datos-accessdesde-visual-basic-475315. 